\title{
Atividade larvicida de extratos de Diplodia pinea frente à Aedes aegypti
}

\author{
Larvicidal activity of Diplodia pinea extracts against Aedes aegypti \\ Actividad larvicida de extractos de Diplodia pinea contra Aedes aegypti
}

Recebido: 23/01/2021 | Revisado: 24/01/2021 | Aceito: 26/01/2021 | Publicado: 04/02/2021

Paula Francislaine Moura

ORCID: https://orcid.org/0000-0003-1617-3144 Universidade Federal do Paraná, Brasil

E-mail: paulafrancislaine19@gmail.com

Fernando Cesar Martins Betim

ORCID: https://orcid.org/0000-0002-1668-8626 Universidade Federal do Paraná, Brasil

E-mail: fernandobetim@hotmail.com

Camila Freitas de Oliveira

ORCID: https://orcid.org/0000-0002-8549-5182

Universidade Estadual do Centro Oeste, Brasil

E-mail: camilafreoli@gmail.com

Josiane de Fátima Gaspari Dias

ORCID: https://orcid.org/0000-0002-8548-8505 Universidade Federal do Paraná, Brasil

E-mail: josianefgdias@gmail.com

Deise Prehs Montrucchio

ORCID: https://orcid.org/0000-0003-1440-7007 Universidade Federal do Paraná, Brasil

E-mail: deisepm@yahoo.com.br

Obdulio Gomes Miguel

ORCID: https://orcid.org/0000-0002-2231-9130

Universidade Federal do Paraná, Brasil

E-mail: obdulio@ufpr.br

Celso Garcia Auer

ORCID: https://orcid.org/0000-0002-4916-2460 Empresa Brasileira de Pesquisa Agropecuária Florestas, Brasil

E-mail: celso.auer@gmail.com

Marilis Dallarmi Miguel

ORCID: https://orcid.org/0000-0002-1126-9211 Universidade Federal do Paraná, Brasil E-mail: marilisdmiguel@gmail.com

\begin{abstract}
Resumo
Inúmeros metabólitos primários e secundários provenientes de fungos tem-se destacado na pesquisa devido o potencial biológico de suas moléculas, com aplicação direta na área da saúde. A dengue é uma patologia transmitida por um vetor, o mosquito Aedes aegypti, e possui grande relevância epidemiológica em diversos países, incluindo o Brasil. Como estratégias do Ministério da Saúde para o combate e controle da dengue emprega-se o uso de inseticidas, eliminação de criadouros e campanhas de conscientização. O uso de compostos inseticidas tem mecanismo de ação sobre o mosquito A. aegypti e suas larvas. Neste contexto e levando em consideração que larvicidas de origem natural, em sua maior parte, não causam impactos tão severos ao meio ambiente e à saúde humana, o objetivo do presente trabalho foi testar a atividade larvicida de extratos obtidos do fungo Diplodia pinea, observar o rendimento destes extratos e realizar um screening químico qualitativo para observação de compostos secundários. Os extratos hexano e clorofórmio apresentaram atividade larvicida significativa com $\mathrm{LC}_{50} 441.42$ e $\mathrm{LC}_{50} 90.49$, respectivamente. A triagem de metabólitos indicou a presença para as classes esteroides, triterpenos e compostos fenólicos e o rendimento dos extratos foi de 1,67 a $47,33 \%$. Estes resultados demonstram que os extratos obtidos a partir do fungo D.pinea apresentam potencial efeito larvicida e ausência de toxicidade frente à Artemia salina.
\end{abstract}

Palavras-chave: Fungo; Dengue; Vetor; Inseticida; Artemia salina; Metabólitos secundários. 


\begin{abstract}
Numerous primary and secondary metabolites from fungi have been highlighted in the research due to the biological potential of their molecules, with direct application in the health area. Dengue is a pathology transmitted by a vector, the mosquito Aedes aegypti, and has great epidemiological relevance in several countries, including Brazil. As strategies of the Ministry of Health to combat and control dengue, the use of insecticides, elimination of breeding sites and awareness campaigns are used. The use of insecticidal compounds has a mechanism of action on the A. aegypti mosquito and its larvae. In this context and taking into account that larvicides of natural origin, in the most part, do not cause such severe impacts to the environment and human health, the objective of this work was to test the larvicidal activity of extracts obtained from the fungus Diplodia pinea, observe the yield of these extracts and perform a qualitative chemical screening to observe secondary compounds. The hexane and chloroform extracts showed significant larvicidal activity with $\mathrm{LC}_{50} 441.42$ and $\mathrm{LC}_{50} 90.49$, respectively. The screening of metabolites indicated the presence for the steroid classes, triterpenes and phenolic compounds and the extracts yield was from 1.67 to $47.33 \%$. These results demonstrate that the extracts obtained from the fungus D.pinea have a potential larvicidal effect and absence of toxicity against Artemia salina.
\end{abstract}

Keywords: Fungus; Dengue; Vector; Insecticide; Artemia salina; Secondary metabolites.

\title{
Resumen
}

Numerosos metabolitos primarios y secundarios de hongos se han destacado en la investigación por el potencial biológico de sus moléculas, con aplicación directa en el área de la salud. El dengue es una patología transmitida por un vector, el mosquito Aedes aegypti, y tiene gran relevancia epidemiológica en varios países, incluido Brasil. Como estrategias del Ministerio de Salud para combatir y controlar el dengue se utiliza el uso de insecticidas, eliminación de criaderos y campañas de concientización. El uso de compuestos insecticidas tiene un mecanismo de acción sobre el mosquito A. aegypti y sus larvas. En este contexto y teniendo en cuenta que los larvicidas de origen natural, en su mayor parte, no ocasionan impactos tan severos al medio ambiente y la salud humana, el objetivo del presente trabajo fue probar la actividad larvicida de extractos obtenidos del hongo Diplodia pinea, observar el rendimiento de estos extractos y realizar un cribado químico cualitativo para observar compuestos secundarios. Los extractos de hexano y cloroformo mostraron una actividad larvicida significativa con $\mathrm{CL}_{50} 441,42$ y $\mathrm{CL}_{50} 90,49$, respectivamente. El cribado de metabolitos indicó la presencia de las clases de esteroides, triterpenos y compuestos fenólicos y el rendimiento de extractos fue de 1,67 a 47,33\%. Estos resultados demuestran que los extractos obtenidos del hongo D.pinea tienen un potencial efecto larvicida y ausencia de toxicidad frente a Artemia salina.

Palabras clave: Hongo; Dengue; Vector; Insecticida; Artemia salina; Metabolitos secundarios.

\section{Introdução}

Os mosquitos do gênero Aedes fazem parte de uma classe relevante de vetores por serem transmissores de patologias importantes na área da saúde. Arboviroses são doenças causadas por arbovírus, que incluem o vírus da dengue, febre chikungunya, Zika vírus e febre amarela. Por esse motivo, as estratégias de controle destes mosquitos (Aedes aegypti e Aedes albopictus) são consideradas importantes em países com alta prevalência dessas doenças, como ocorre no Brasil. O Aedes aegypti apresenta disseminação e sobrevivência em ambientes urbanos, tendo como criadouros principais os reservatórios de água parada. Isso favorece potencialmente a rápida proliferação da espécie devido à possibilidade de reprodução e disponibilidade de fontes de alimentação (Zara et al., 2016).

A dengue possui grande relevância epidemiológica em regiões tropicais e subtropicais da África e Ásia, e em países da América como o Brasil. O vírus da dengue pertence ao gênero Flavivirus e à família Flaviviridae, apresentando quatro sorotipos denominados DEN-1, DEN-2, DEN-3, DEN-4, sendo transmitido por mosquitos fêmeas principalmente da espécie $A$. aegypti. A dengue clássica tem evolução normalmente benigna e como sintomas mais comuns podem-se citar febre alta (39 a $40{ }^{\circ} \mathrm{C}$ ), cefaléia, cansaço, mialgia e fadiga. No caso da dengue hemorrágica, ocorrem alterações na permeabilidade vascular e cascata de coagulação sanguínea. Nos casos de complicações, a dengue pode evoluir para o choque hipovolêmico e óbito, sendo essa considerada a forma mais grave da doença (Furtado et al., 2019; Ortega et al., 2015).

No Brasil, estudos apontaram que a partir da década de 90 houve expansão da circulação do vírus para cerca de 20 estados brasileiros, o que acarretou em um aumento da disseminação da dengue. Em estudo publicado, Furtado et al., (2019) relataram que no ano de 2015, 1.649 .008 de casos foram registrados no país. Outros estudos como de Rickli et al., (2020) trouxeram dados atualizados da Organização Mundial da Saúde - OMS e Organização Pan-Americana de Saúde - OPAS 
citando que, no ano de 2020, 1.600 .000 milhões de casos de dengue, 37.279 casos de chikungunya e 7.452 casos de Zika vírus foram notificados nas Américas. Neste contexto, o Brasil representa $65 \%$ dos casos de dengue, o que o torna o país com a maior prevalência da doença no continente americano (WHO/OMS, 2020; Rickli et al., 2020).

Devido à alta prevalência da dengue no Brasil, desde o século XX há programas de controle da doença que incluem o controle do vetor por diferentes métodos. Dentre os métodos citam-se os mecânicos que compreendem a eliminação de criadouros e uso de telas de proteção em janelas. No caso dos métodos biológicos, emprega-se o uso de predadores como fungos e bactérias, que liberam bioativos como as toxinas. Nos métodos químicos, se faz uso de inseticidas ou larvicidas (Braga \& Valle, 2007; Brasil, 2009; Zara et al., 2016).

O mosquito A. aegypti é um artrópode da família Diptera, Culicidae e gênero Aedes, descrito pela primeira vez no Egito no ano de 1762. No Brasil, suspeita-se que sua introdução tenha sido entre os séculos XVI e XIX. Com a destruição de muitos habitats naturais e a boa adaptação do mosquito em água parada em ambientes silvestres, rurais e urbanos com grandes populações, a partir do século XX foi notória a intensificação da disseminação desse mosquito em vários estados do país (Beserra et al., 2009; Zara et al., 2016).

Inseticidas são resumidamente substâncias químicas ou biológicas utilizadas para controlar insetos danosos seja no seu estado larval ou adulto. Para isso, além de toxicidade frente ao agente agressor, os inseticidas devem preferencialmente apresentar eficácia em baixas concentrações, baixa toxicidade em mamíferos e animais superiores, ausência de fitotoxicidade e biodegradabilidade. Podem-se citar duas classes de inseticidas, os químicos e biológicos que são capazes de interferir no processo de desenvolvimento larval do vetor (Garcez et al., 2013).

Desde a década de 40, inseticidas advindos de fontes naturais vêm sendo utilizados, com seu uso intensificado ao longo dos anos. Dentre os inseticidas empregados em combate de pragas, podem-se citar, por exemplo, os extraídos de plantas como o alcaloide nicotina, isolado de espécies de Nicotiana, piretroides (piretrina e aletrina) extraídos do Chrysanthemum cinerariaefolium e o limonoide azadiractina obtido da Azadirachta indica (Hollingworth, 1976). Algumas pesquisas relatam o uso de inseticidas extraídos de fungos patogênicos como o Chrysosporium tropicum, demonstrado no trabalho de Soni \& Prakash (2012).

Entre as décadas de 50 a 70, os inseticidas químicos foram amplamente utilizados, como os organoclorados, organofosforados, carbamatos e piretróides. Posteriormente, se observou que o uso destas substâncias impactava negativamente e de maneira indefinida na fauna, flora e meio ambiente, o que aumentou o interesse e a retomada de pesquisas com inseticidas de origem natural (Braga \& Valle, 2007; Garcez et al., 2013).

O fungo Diplodia pinea é um fitopatógeno que causa doenças em coníferas e está amplamente distribuído pelo mundo (Basilio, 2013). Vários fungos têm produzido micotoxinas que podem causar problemas no desenvolvimento de outros organismos (Ciegler, 1977). Beard \& Walton (1969) demonstraram o efeito tóxico do ácido kojico isolado de Aspergillus flavus var. columnaris sobre larvas de Aedes atropalpus. No caso de insetos, Ciegler (1977), em sua revisão sobre micotoxinas discute a possibilidade de uso dessas substâncias e seus derivados contra insetos vetores, mencionando a diplodiatoxina produzida por Diplodia maydis. Este fungo estudado por Spiassi et al., (2015) também revelou um potencial efeito alelopático em plantas. Assim, a pesquisa de outros fungos do mesmo gênero, como $D$. pinea, pode revelar um potencial efeito inseticida e larvicida.

Com base em informações na literatura sobre fungos com atividade inseticida, o objetivo do presente estudo foi avaliar a atividade larvicida e toxicidade ambiental de extratos de D. pinea frente a larvas de A. aegypti, o rendimento dos extratos empregados nos testes biológicos e realizar um screening por cromatografia em camada delgada (CCD) para indicar possíveis classes de metabólitos secundários presentes nos extratos do micélio e caldo de cultivo do fungo. 


\section{Metodologia}

O isolado de $D$. pinea desse estudo foi obtido de um indivíduo infectado de Pinus taeda (27046'18"S; 51³6'14"W) e se encontra preservado em frascos de Castellani a $4{ }^{\circ} \mathrm{C}$ na Coleção de Fungos e Oomicetos Florestais da Embrapa Florestas, Colombo/PR, Brasil. Uma amostra do fungo está depositada no herbário da Universidade Estadual do Oeste do Paraná UNIOESTE, exsicata UNOP 4242 e a presente pesquisa possui autorização do SISGEN nº A80029B.

Para a produção in vitro de culturas puras do fungo, o mesmo foi repicado em placas de Petri contendo meio batata dextrose ágar (BDA). As placas foram incubadas em estufa BOD a $24{ }^{\circ} \mathrm{C}$, no escuro, por 10 dias (Silva, 2018 adaptado). Posteriormente, dois discos de micélio ágar de cultura pura do fungo foram inoculados em frascos de vidro contendo $50 \mathrm{~mL}$ de caldo batata dextrose - BD (meio comercial BD Kasvi®). O caldo foi previamente esterilizado em autoclave e as inoculações e repiques ocorreram em fluxo laminar para que não houvesse contaminação. Os frascos de vidro contendo o caldo BD e o fungo foram acondicionados em estufa BOD a $16^{\circ} \mathrm{C}$, no escuro, por 7 dias, sem aeração mecânica (Moura, 2017). Após a retirada das culturas, as mesmas foram separadas do caldo de cultivo por filtração em fluxo laminar sendo observadas características como odor, cor e turbidez, para garantia da pureza do inóculo. A massa fúngica foi congelada em freezer e liofilizada (5 dias a -80 ${ }^{\circ} \mathrm{C}$ ) e o caldo de cultivo foi concentrado em rotaevaporador a $50{ }^{\circ} \mathrm{C}$ até redução de $95 \%$ do seu volume inicial.

Os extratos utilizados neste estudo foram produzidos empregando o aparelho de Soxhlet modificado (Patente INPI 0601703-7 A). Foram utilizados para obtenção dos extratos do micélio (30 g) os solventes em polaridade crescente n-hexano, clorofórmio, acetato de etila, metanol e uma mistura de etanol/água 1:1 (v/v), cada extração foi realizada por 72 h. Para obtenção do extrato a partir do caldo de cultivo concentrado $(200 \mathrm{~mL})$ foi empregado o solvente acetato de etila utilizando o mesmo equipamento por 72 h (Carvalho, 2009). Após secagem dos extratos, o rendimento de cada um foi calculado quantitativamente.

Os extratos foram denominados de acordo com seu solvente de extração hexano do micélio fúngico (EH); clorofórmio do micélio fúngico; acetato de etila do micélio fúngico (EAE); metanol do micélio fúngico (EM); etanol/água do micélio fúngico (EEA) e acetato de etila do meio de cultivo (EAEM). Os extratos foram submetidos a um screening químico qualitativo por cromatografia em camada delgada $(\mathrm{CCD})$ e à avaliação da sua atividade frente larvas de A. aegypti e potencial tóxico preliminar frente à Artemia salina.

No ensaio de CCD, todos os extratos foram solubilizados em metanol e aplicados sobre cromatoplacas com o auxílio de um capilar. Estas foram submetidas à eluição com diferentes fases móveis, e reveladas para a identificação das classes de metabólitos secundários. Para a pesquisa de esteroides e triterpenos, foi utilizada a fase móvel tolueno:acetato de etila 93:7(v/v) e como revelador a vanilina sulfúrica 1\% (Wagner, 1996). Na pesquisa dos compostos fenólicos foi empregada a fase móvel acetato de etila:ácidofórmico:ácido acético glacial:água 100:11:11:26(v/v/v/v) e os reveladores NEU e cloreto férrico (Wagner, 1996). Quanto à pesquisa de alcaloides foi aplicada como fase móvel clorofórmio:metanol 95:5 (v/v) e o revelador de Dragendorff (Wagner, 1996).

A metodologia empregada para avaliação quantitativa da atividade larvicida foi adaptada de Garcez et al., (2013) e Betim et al., (2019). Para a avaliação da atividade dos extratos, ovos de A. aegypti (cepa Rockefeller cedida pela Fundação Oswaldo Cruz) foram eclodidos em laboratório a $25 \pm 3{ }^{\circ} \mathrm{C}$, umidade relativa de $80 \%$, em estufa BOD, e alimentados com ração para peixes (Alcon basic, complexo MEP 200) até o terceiro estágio de desenvolvimento larval. Os extratos (amostras) foram diluídos em 0,5\% de dimetilsulfóxido (DMSO) e foram empregadas concentrações de 1000,100 e $10 \mu \mathrm{g} \cdot \mathrm{mL}{ }^{-1}$. Após a eclosão, 10 larvas foram tratadas com água, DMSO e com os extratos de $D$. pinea por 24 h. Posteriormente, as larvas vivas e mortas foram contadas para cada tratamento. O teste foi conduzido em triplicata (30 larvas para cada concentração de amostra). 
O método probito foi usado para determinar os valores da concentração letal ( $\mathrm{LC}_{50}$ e $\mathrm{LC}_{90}$ ), que corresponde a intervalos de confiança de $95 \%$. O teste do qui-quadrado também foi realizado para o ensaio. Todas as análises estatísticas foram realizadas usando IBM SPSS Statistics versão 20.0 (IBM Corp., Armonk, NY, EUA).

Com a finalidade de realizar uma triagem preliminar quanto à segurança ambiental dos extratos que apresentaram atividade larvicida, empregou-se o teste de toxicidade frente à Artemia salina. Os ovos de A. salina (200 mg/400 mL) foram colocados em água do mar artificial (38 g de sal marinho Blue Greasure® em $1000 \mathrm{~mL}$ de água destilada) por 48 h, em estufa BOD com fotoperíodo de $12 \mathrm{~h}$, sob aeração contínua e controlada temperatura entre 27 e $30^{\circ} \mathrm{C}$, para eclodir. Os extratos foram diluídos em metanol e o solvente evaporado em estufa a $40{ }^{\circ} \mathrm{C}$ por $24 \mathrm{~h}$. Como controle positivo foi utilizado o SDS (dodecil sulfato de sódio) e o metanol foi empregado como controle negativo. Após a eclosão dos ovos, 10 larvas de A. salina foram transferidas para frascos de vidro com capacidade de $10 \mathrm{~mL}$ contendo extratos ressuspensos com $5 \mathrm{~mL}$ de solução de água do mar artificial nas concentrações de 10, 100, 250, 500 e $1000 \mu \mathrm{g} \cdot \mathrm{mL}^{-1}$. Os mesmos procedimentos foram realizados com os controles positivo e negativo. O teste foi realizado em triplicata para todas as amostras e controles. As larvas mortas e vivas foram contadas após 24h (Meyer et al., 1982). Os dados foram analisados pelo método estatístico IBM SPSS 22, possibilitando a determinação dos valores de $\mathrm{LC}_{50}$. As frações consideradas ativas foram aquelas com valor de $\mathrm{CL}_{50}$ abaixo de 1000 ppm.

\section{Resultados e Discussão}

Os frascos de cultivo contendo $D$. pinea apresentaram aspecto satisfatório e condizente com o esperado para um cultivo sem contaminação. Não foram observados alteração de odor, turbidez do caldo de cultivo tampouco na coloração do caldo e do micélio fúngico.

Após o processo de liofilização do micélio e concentração do caldo de cultivo, obteve-se 30 g e $200 \mathrm{~mL}$, respectivamente, que foram submetidos ao processo de extração.

Após de evaporado todo o solvente de extração, foi verificado o peso em gramas para o cálculo do rendimento de cada extrato conforme pode ser observado na Tabela 1.

Tabela 1 - Rendimento dos extratos de micélio e caldo de cultivo de Diplodia pinea após secagem.

\begin{tabular}{ccc}
\hline Extratos & Massa final (g) & Rendimento (\%) \\
\hline Extrato Hexano (micélio) & 0,82 & 2,73 \\
\hline Extrato Clorofórmio (micélio) & 0,90 & 3 \\
\hline Extrato Acetato de Etila (micélio) & 0,50 & 1,67 \\
\hline Extrato Metanol (micélio) & 14,2 & 47,33 \\
\hline Extrato Etanol/água 1:1 (v/v) (micélio) & 2,75 & 9,16 \\
\hline $\begin{array}{c}\text { Extrato Acetato de Etila } \\
\text { (caldo de cultivo) }\end{array}$ & 0,50 & \multirow{2}{*}{1,67} \\
\hline
\end{tabular}

Fonte: Autores (2021).

Observou-se que os extratos de maior rendimento foram os obtidos com emprego dos solventes metanol e etanol/água 1:1 (v/v). Isso provavelmente se deve ao fato de solventes alcoólicos extraírem inúmeras substâncias de diferentes grupos químicos devido a sua polaridade (Pandey \& Tipathi, 2014; Rodrigues et al., 2016).

No screening químico dos extratos de D. pinea, observou-se positividade para esteroides, triterpenos, compostos fenólicos, em todos os extratos. Não foi observada a presença de alcaloides. Estes resultados podem auxiliar na pesquisa de metabólitos, relacionando-os com atividades biológicas. 
Quanto ao potencial larvicida, as amostras que demonstraram atividade foram os extratos hexano e clorofórmio do micélio de $D$. pinea (Tabela 2), devido ao seu índice de mortalidade considerável e uma boa taxa de LC 50 para larvas do $A$. aegypti.

Tabela 2 - Mortalidade e concentração letal dos extratos de Diplodia pinea frente a larvas de Aedes aegypti.

\begin{tabular}{|c|c|c|c|c|c|c|}
\hline Extrato & $\begin{array}{c}\text { Concentração } \\
\left(\mu \mathrm{g} \cdot \mathrm{mL}^{-1}\right)\end{array}$ & $\begin{array}{c}\text { Mortalidade } \\
(\%) \pm \text { SD }\end{array}$ & $\begin{array}{c}\text { LC }_{50}\left(\boldsymbol{\mu g} \cdot \mathrm{mL}^{-1}\right) \\
\text { (LCMin-LCMáx) }\end{array}$ & $\begin{array}{c}\text { LC90 }\left(\mu \mathrm{g} . \mathrm{mL}^{-1}\right) \\
\text { (LCMin-LCMáx) }\end{array}$ & $\mathbf{x}^{2}$ & (df) \\
\hline \multirow{3}{*}{$\mathrm{EH}$} & 10 & $6.66 \pm 0.57$ & & \multirow{3}{*}{$>1000$} & \multirow{3}{*}{1.76} & \multirow{3}{*}{1} \\
\hline & 100 & $26.66 \pm 0.57$ & 441.42 & & & \\
\hline & 1000 & $63.33 \pm 0.57$ & $(209.26-1429.68)$ & & & \\
\hline \multirow{3}{*}{$\mathrm{EC}$} & 10 & $23.33 \pm 0.57$ & & \multirow{3}{*}{$>1000$} & \multirow{3}{*}{1.09} & \multirow{3}{*}{1} \\
\hline & 100 & $53.33 \pm 0.57$ & 90.49 & & & \\
\hline & 1000 & $76.66 \pm 0.57$ & $(32.60-238.87)$ & & & \\
\hline \multirow{3}{*}{ EAE } & 10 & $6.66 \pm 0.57$ & \multirow{3}{*}{$>1000$} & \multirow{3}{*}{$>1000$} & \multirow{3}{*}{ n.a } & \multirow{3}{*}{ n.a } \\
\hline & 100 & $20.0 \pm 1.00$ & & & & \\
\hline & 1000 & $36.66+1.15$ & & & & \\
\hline \multirow{3}{*}{ EM } & 10 & $0.0 \pm 0.00$ & \multirow{3}{*}{$>1000$} & \multirow{3}{*}{$>1000$} & \multirow{3}{*}{ n.a } & \multirow{3}{*}{ n.a } \\
\hline & 100 & $3.33 \pm 0.57$ & & & & \\
\hline & 1000 & $16.66 \pm 0.57$ & & & & \\
\hline \multirow{3}{*}{ EEA } & 10 & $0.0 \pm 0.00$ & \multirow{3}{*}{$>1000$} & \multirow{3}{*}{$>1000$} & \multirow{3}{*}{ n.a } & \multirow{3}{*}{ n.a } \\
\hline & 100 & $0.0 \pm 0.00$ & & & & \\
\hline & 1000 & $3.33+0.57$ & & & & \\
\hline \multirow{3}{*}{ EAEM } & 10 & $0.0 \pm 0.00$ & \multirow{3}{*}{$>1000$} & \multirow{3}{*}{$>1000$} & \multirow{3}{*}{ n.a } & \multirow{3}{*}{ n.a } \\
\hline & 100 & $6.66 \pm 0.57$ & & & & \\
\hline & 1000 & $20.0 \pm 1.00$ & & & & \\
\hline
\end{tabular}

Legenda: Extrato hexano do micélio fúngico (EH); Extrato clorofórmio do micélio fúngico (EC); Extrato acetato de etila do micélio fúngico (EAE); Extrato Metanol do micélio fúngico (EM); Extrato Etanol/água do micélio fúngico (EEA); Extrato acetato de etila do meio de cultivo (EAEM). Limite de confiança entre a concentração mínima e máxima de extrato para atividade larvicida (LCMin - LCMáx). $x^{2}=$ teste estatístico qui-quadrado. (df) = grau de liberdade. Não se aplica (n.a).

Nota: não foi observada mortalidade no controle negativo; para o controle positivo a mortalidade foi de $100 \%$ das larvas. Intervalo de confiança igual ou superior a $95 \%$.

Fonte: Autores (2021).

A atividade mais efetiva foi observada no extrato clorofórmio, que apresentou uma ótima $\mathrm{LC}_{50}$ de 90.49 e uma excelente taxa de mortalidade mesmo na menor concentração testada $\left(10 \mu \mathrm{g} \cdot \mathrm{mL}^{-1}\right)$.

Segundo uma revisão realizada por Pinheiro et al., (2020), inúmeros fungos apresentam atividade larvicida frente a diferentes vetores, porém não se observou estudos dessa atividade para a espécie D. pinea. Em um estudo realizado por Sathiyanathan e Umarajan (2019) o fungo Aspergillus terreus apresentou excelente atividade larvicida frente à Anopheles stephensis e Culex quinquefasciatus. Uma pesquisa realizada por Geris et al., (2008), vincula a atividade larvicida de extratos fúngicos obtidos de Penicillium, aos meroterpenóides presentes (Geris et al., 2008; Sathiyanathan \& Umarajan, 2019; Pinheiro, et al., 2020). Em pesquisa publicada em 2008 por Pandi e colaboradores, Botryodiplodia theobromae, fungo da mesma família de D. pinea (Botryosphaeriaceae), apresentou atividade larvicida frente a $C$. quinquefasciatus em seu segundo estágio nas primeiras $24 \mathrm{~h}$ (Pandi et al., 2008).

Bücker e colaboradores (2013) comprovaram a atividade larvicida de extratos do micélio de Pestalotiopsis virgulata, frente a larvas de Aedes aegypti e Anopheles larvae. Estudos de Podder \& Grosh (2019) demonstraram que fungos do gênero Trichoderma como T. asperellum, além de serem utilizados como promotores de crescimento vegetal na área agrícola têm aplicabilidade como agente de controle biológico por apresentar atividade larvicida contra o mosquito Anopheles, vetor da malária (Podder \& Grosh, 2019). 
O ensaio de A. salina é um teste preliminar de toxicidade biológica in vitro, importante para medir o potencial tóxico de uma substância ou extrato. Pode ser utilizado em estudos com extratos que apresentam atividades biológicas com a finalidade de confirmar ou não sua toxicidade ambiental. Segundo Amarante et al., 2011 e Lima et al., 2011 uma amostra com $\mathrm{CL}_{50}$ acima de $1000 \mu \mathrm{g} / \mathrm{mL}$ tem ausência de toxicidade. Os extratos hexano e clorofórmio de $D$. pinea não apresentaram toxicidade para este teste em nenhuma das concentrações testadas, pois apresentaram uma $\mathrm{CL}_{50}$ superior a $1000 \mu \mathrm{g} / \mathrm{mL}$.

Larvicidas obtidos de fontes naturais representam uma opção biotecnológica menos tóxica ao meio ambiente e à saúde humana e animal (Ragavendran_et al., 2019). Os fungos são reconhecidamente fontes de substâncias bioativas que apresentam diferentes aplicabilidades. Devido à importância do desenvolvimento de larvicidas de origem natural, ressalta-se a importância de mais pesquisas de biocompostos extraídos de fungos, por sua potencial aplicabilidade na área de saúde humana, veterinária e agrícola.

\section{Conclusão}

Observou-se que para a produção dos extratos, a partir do fungo $D$. pinea, houve melhor rendimento com o solvente metanol. No screening químico qualitativo, os extratos apresentaram positividade para esteroides, triterpenos e compostos fenólicos.

Foi observada uma taxa de mortalidade significativa para larvas de A. aegypti quando as mesmas foram submetidas aos extratos hexano $\mathrm{LC}_{50}$ de 441,42 e clorofórmio $\mathrm{LC}_{50}$ de 90,41. Frente a estes resultados, sugere-se que o fungo $D$. pinea possui potencial larvicida e não apresentou toxicidade em teste preliminar.

Frente aos resultados obtidos, os autores sugerem pesquisas futuras e continuidade de estudos com fungos fitopatogênicos.

\section{Agradecimentos}

Este trabalho foi realizado com o apoio da Coordenação de Aperfeiçoamento de Pessoal de Nível Superior - Brasil (CAPES) - Código Financiador 001. Os autores agradecem à Embrapa Florestas, CNPq e Universidade Federal do Paraná pelo apoio financeiro e bolsas. Os autores declaram que não há conflito de interesses.

\section{Referências}

Amarante C. B., Muller A. H., Povoa M. M., \& Dolabela M. F. (2011). Estudo fitoquímico biomonitorado pelos ensaios de toxicidade frente à Artemia salina e de atividade antiplasmódica do caule de aninga (Montrichardia linifera). Acta Amazonica 41(3), 431-434. https://doi.org/10.1590/S004459672011000300015 .

Beard, R. L., \& Walton, G. S. (1969). Kojic acid as an insecticidal mycotoxin, Journal of Invertebrate Pathology, 14(1), 53-59. https://doi.org/10.1016/00222011(69)90010-X.

Basilio, P. R. R. C. (2013). Caracterização morfofisiológica, patogênica e molecular de isolados de Diplodia pinea. Tese de Doutorado. Universidade Federal do Paraná.

Beserra, E. B., Freitas, E. M., Souza, J. T., Fernandes, C. R. M., \& Santos K. D. (2009). Ciclo de vida de Aedes (Stegomyia) aegypti (Diptera, Culicidae) em águas com diferentes características. Iheringia, Série Zoologia 99(3), 281-285. https://www.scielo.br/pdf/isz/v99n3/08.pdf

Braga, I. A., \& Valle, D. (2007). Aedes aegypti: inseticidas, mecanismos de ação e resistência. Epidemiologia e Serviços de Saúde 16(4), 279-293. http://dx.doi.org/10.5123/S1679-49742007000400006.

Brasil. Ministério da Saúde. (2009). Secretaria de Vigilância em Saúde. Departamento de Vigilância Epidemiológica. Diretrizes nacionais para a prevenção e

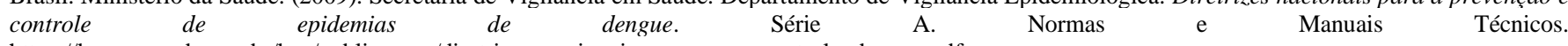
https://bvsms.saude.gov.br/bvs/publicacoes/diretrizes_nacionais_prevencao_controle_dengue.pdf. 
Bücker, A., Bücker, N. C. F., Souza, A. Q. L., Gama, A. M., Rodrigues-Filho, E., Costa, F. M., Nunez, C. V., Silva, A. C., \& Tadei, W. P. (2013). Larvicidal effects of endophytic and basidiomycete fungus extracts on Aedes and Anopheles larvae (Diptera, Culicidae). Revista da Sociedade Brasileira de Medicina Tropical 46(4), 411-419. https://doi.org/10.1590/0037-8682-0063-2013.

Carvalho, J. L. S.; Cunico, M. M.; Dias, J. F. G; Miguel, M. D.; \& Miguel, O. G. (2009). Termoestabilidade de processos extrativos de Nasturtium officinale R. Br., brassicaceae por sistema de Soxhlet modificado. Química Nova 32(4), 1031-1035. doi: https://doi.org/10.1590/S0100-40422009000400034

Ciegler, A. (1977). Mycotoxins as insecticides. The saprophytic and aerobic bacteria and fungi. Conference Report. Biological Regulation of Vectors 135-144. https://books.google.com.br/books?hl=pt-BR\&lr=\&id=os9KfRcp9HIC\&oi=fnd\&pg=PA135\&dq=diplodia+insecticide\&ots=nXLeuB818P\&sig=hKw4PrPtA VuCQvE6QyKfHzzqMHQ\&redir_esc=y\#v=onepage\&q\&f=false.

Furtado, A. N. R., Lima, A. S. F., Oliveira, A. S., Teixeira, A. B., Ferreira, D. S., Oliveira, E. C., Cavalcanti, G. B., Sousa, W. A., \& Lima, W. M. (2019). Dengue e seus avanços. Revista Brasileira de Análises Clínicas 51(3), 196-201. doi: 10.21877/2448-3877.201900723.

Garcez, W. S., Garcez, F. R., Silva, L. M. G. E., \& Sarmento, U. C. (2013). Substâncias de origem vegetal com atividade larvicida contra Aedes aegypti. Revista Virtual de Química 5(3), 363-393. 10.5935/1984-6835.20130034.

Geris, R., Rodrigues-Fo, E., Silva, H. H. G., \& Silva, I. G. (2008). Larvicidal effects of fungal meroterpenoids in the control of Aedes aegypti L., the main vector of dengue and yellow fever. Chemistry \& Biodiversity 5(2), $341-345$. https://doi.org/10.1002/cbdv.200890032

Hollingworth, R. M. (1976). Chemistry, biological activity, and uses of formamidine pesticides. Environ Health Perspect 57-69. 10.1289/ehp.761457.

Lima, C. P., Cunico, M. M., Trevisan, R. R., Philippsen, A. F., Miguel, O. G., \& Miguel, M. D. (2011). Efeito alelopático e toxicidade frente à Artemia salina Leach dos extratos do fruto de Euterpe edulis Martius. Acta Botanica Brasilica 25(2), 331-336. https://doi.org/10.1590/S0102-33062011000200009

Moura, P. F. (2017). Caracterização e otimização dos métodos de cultivo dos fungos Armillaria sp., Botrytis cinerea e Diplodia pinea, estudo químico e avaliação das atividades biológicas de extratos de Diplodia pinea. Dissertação de Mestrado. Universidade Federal do Paraná.

Meyer B. N., Ferrigni N. R., Putnam J. E., Jacobsen L. B., Nichols D. E., \& Mclaughlin J. L. (1982). Brine shrimp: a convenient general bioassay for active plant constituents. Planta Medica 45, 31-34. doi: 10.1055/s-2007-971236.

Ortega, M. R. O., Moreno, M. L. C. C., \& Domígues, M. L. A. D. (2015). Análisis sobre el dengue, su agente transmisor y estrategias de prevención y control Archivo Médico de Camagüey 19(2), 189-202. https://www.medigraphic.com/pdfs/medicocamaguey/amc-2015/amc152m.pdf

Pandey, A.; \& Tripathi, S. (2014). Concept of standardization, extraction and prephytochemical screening strategies for herbal drug. Journal of Pharmacognosy Phytochemistry 2(5), 115-119. https://www.phytojournal.com/vol2Issue5/Issue_jan_2014/11.pdf

Pandi, M., Koodalingam, A., Manikandan, R., \& Muthumary, J. (2008). Larvicidal activity of two extracts of endophitic fungi isolated from Pongamiapinata (L) Pierre. Indian Journal of Applied Microbiology 8(1), 34-36. http://www.ijamicro.com/8-1-2008/8.pdf.

Pinheiro, J. B., Polonio, J. C., Orlandelli, R. C., Pamphile, J. A., \& Golias, H. C. (2020). Atividade larvicida de fungos endofíticos: uma revisão. Brazilian Journal of Development 6(6), 35761-35774. 10.34117/bjdv6n6-205.

Podder, D. \& Grosh, S. K. (2019). A new application of Trichoderma asperellum as an anopheline larvicide for ecofriendly management in medical science. Scientific Reports 9(Article 1108), 1-15. doi: https://doi.org/10.1038/s41598-018-37108-2.

Rickli, M. E., Pradella, H. Q., Gomes, M. G., Belini, B. P., Bortolucci, W. C., Fernandez, C. M. M., Colauto, N. B., Linde, G. A., \& Gazim, Z. C. (2020). Atividade bioinseticida de Philodendron bipinnatifidum. Research, Society and Development 9(11), 1-12. doi: http://dx.doi.org/10.33448/rsd-v9i11.10464.

Rodrigues, F. A., Pimenta, V. S. C., Braga, K. M. S., \& Araújo, E. G. (2016). Obtenção de extratos de plantas do cerrado. Enciclopédia Bioesfera 13(23), 870888. 10.18677/Enciclopedia_Biosfera_2016_075

Sathiyanathan, M. \& Umarajan, K. M. (2019). Larvicidal activity of endophytic fungi isolated from selected medicinal plants on Aedes aegypti. Journal of Pharmacognosy and Phytochemistry 8(2), 247 - 253. https://www.phytojournal.com/archives/2019/vol8issue2/PartE/8-1-219-523.pdf

Silva, F. B., Mazarotto, E. J., Santos, A. F., \& Auer, C. G. (2018). Caracterização morfológica, fisiológica e patogênica de isolados de Armillaria sp. da Região Sul do Brasil. Summa Phytopathologica, 44(1), 23-31. https://doi.org/10.1590/0100-5405/175653.

Soni, N.; \& Prakash, S. (2012). Efficacy of fungus mediated silver and gold nanoparticles against Aedes aegypti larvae. Parasitology Research 110, 175-184. $10.1007 / \mathrm{s} 00436-011-2467-4$.

Spiassi, A., Nóbrega, L. H. P., Rosa, D. M., Pacheco, F. P., Senem, J., \& Lima, G. P. (2015). Allelopathic effects of pathogenic fungi on weed plants of soybean and corn crops. Bioscience journal 31(4), 1037-1048. https://doi.org/10.14393/BJ-v31n4a2015-26142.

Wagner, H. (1996). Plant Drugs analysis. (2a ed.) Berlin: Springer, 298-299 e 319.

World Health Organization - WHO (Organização Mundial da Saúde). (2020). Epidemiological Update: Arbovirus. Washington, D.C.

Zara, A. L. S. A., Santos, S. M., Fernandes-Oliveira, E. S., Carvalho, R. G., \& Coelho, G. E. (2016). Estratégias de controle do Aedes aegypti: uma revisão. Epidemiologia e Serviços de Saúde 26(2), 391-404. http://dx.doi.org/10.5123/S1679-49742016000200017. 